Gynäkologe 2011 · 44:261-263

DOI 10.1007/s00129-010-2701-7

Online publiziert: 27. März 2011

c) Springer-Verlag 2011

\author{
K. Vetter ${ }^{2} \cdot$ W. Jonat ${ }^{1} \cdot$ S. Mundlos ${ }^{3}$ \\ ${ }^{1}$ Klinik für Gynäkologie und Geburtshilfe, Universität Schleswig-Holstein, \\ Campus Kiel \\ ${ }^{2}$ Klinik für Geburtsmedizin, Vivantes-Klinikum Berlin-Neukölln, Berlin \\ ${ }^{3}$ Institut für Medizinische Genetik, Charitè, Universitätsmedizin Berlin, \\ Campus Virchow, Berlin
}

\title{
Humangenetik in der Medizin
}

senszuwachs in diesem dynamischen Gebiet, das uns alle in unserem ärztlichen Alltag angeht.

Die Sequenzierung des menschlichen Genoms 2003 markierte definitiv der Start in eine neue Ära des Blicks in uns Menschen. Auch wenn wir nach wie vor weit davon entfernt sind, seine Informationen zu verstehen, haben wir seither Entwicklungen punktueller Erkenntnisse beobachten können, die uns vor einigen Monaten noch nicht möglich schienen. Die Entwicklung ist extrem dynamisch. Entsprechend gibt es für jeden von uns eine Menge zu lernen.

Dass neue Erkenntnisse nicht nur Macht für den Besitzer der Informationen bedeuten, sondern auch als bedrohlich empfunden werden können, ist nicht neu. Um unter solchen Umständen die Hoheit über die persönlichen Daten abzusichern, wurde 2009 das Gendiagnostikgesetz nach langem Vorlauf eilig beschlossen.

Ärzte unseres Gebiets können danach eine Erlaubnis zur fachgebundenen genetischen Beratung erwerben, deren Grundlage neben kommunikativer Kompetenz insbesondere die Kenntnis moderner Genetik ist.

Das vorliegende Themenheft soll als Basis dieser Kenntnisse moderner Genetik dienen. Die Herausgeber sind Prof. Mundlos und seinem Team für die herausragenden wohlgesetzten Artikel ausgesprochen dankbar und wünschen gleichzeitig den Lesern den erwünschten Wis-

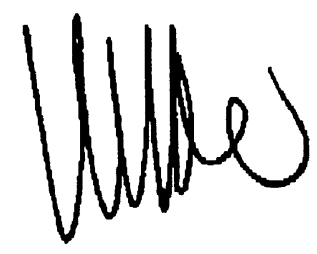

Prof. Dr. Klaus Vetter

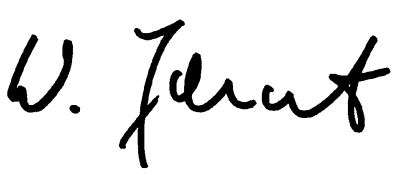

Prof. Dr. Walter Jonat

\section{Humangenetik 2011}

Die Bedeutung der Humangenetik für die medizinische Praxis hat sich über die letzten Jahrzehnte fundamental geändert. Während das Fach in den 7oerund 8oer-Jahren des vergangenen Jahrhunderts noch ein weitgehend zurückgezogenes Dasein führte und sich im Wesentlichen mit der klinischen Beschreibung von extrem seltenen Krankheitsbildern beschäftigte, ist die Humangenetik jetzt zum Mainstream geworden und findet Einzug in verschiedenste Fachgebiete der Medizin. Ganz wesentlich ist diese Veränderung auf zwei technische Neuerungen zurückzuführen: die Einführung der Pränataldiagnostik und das Humangenomprojekt. Die Einführung der genetischen Pränataldiagnostik hat die Bedeutung humangenetischer Untersuchungen ganz wesentlich gestärkt und die Chromosomenanalyse zu einer Routinediagnostik werden lassen. So wichtig die Zytogenetik für die Diagnose von Trisomien und anderen chromosomalen Aberrationen ist, so sehr war auch klar, dass damit nur ein verschwindend kleiner Teil von Veränderungen unseres Genoms erfasst werden kann.

\section{Völlig neue Methoden ermöglichen einen wesentlich umfassenderen Einblick in das Genom}

Mittlerweile haben völlig neue Methoden Einzug gehalten, die uns einen wesentlich umfassenderen Einblick in das Genom ermöglichen. Entscheidend basieren alle Weiterentwicklungen auf dem Humangenomprojekt und der damit verbundenen Sequenzierung des menschlichen Genoms. Mittlerweile sind die im Humangenomprojekt gewonnenen Daten und Erkenntnisse Allgemeingut und für Jeden über öffentlich zugängliche Datenbanken einsehbar. Das Humangenomprojekt hat zu einer Flut von Studien geführt, die sich mit den genetischen Auswirkungen bestimmter genomischer Veränderungen beschäftigen und Korrelationen zwischen Geno- 
typ und Phänotyp herstellen. Die Basis für eine zunehmende Zahl monogener Erkrankungen ist inzwischen aufgeklärt, trotzdem werden immer wieder weitere identifiziert. Für viele häufige polygene Erkrankungen wurden Faktoren entdeckt, welche die Erkrankungswahrscheinlichkeit (sogenannte Suszeptibilitätsfaktoren) erhöhen, die aber einzeln, von wenigen Ausnahmen abgesehen, nur einen geringen Effekt haben und damit wenig prädiktiv sind.

\section{Sequenzvarianten zwischen Individuen treten etwa alle $1000 \mathrm{bp}$ auf und summieren sich zu etwa 3 Millionen}

Zugleich ist die Komplexität des humanen Genoms offenbar geworden, dessen Variabilität zwischen einzelnen Personen nicht nur durch Variationen in der Sequenz, sondern auch durch Variationen in der Kopienzahl deutlich wird. Sequenzvarianten zwischen Individuen treten etwa alle 1000 bp auf und summieren sich zu etwa 3 Mio. Sequenzdifferenzen oder $0,1 \%$ des Genoms (zum Vergleich, die Differenz zum Schimpansen ist nur $10-$ bis 15 -mal so groß). Mindestens ebenso wichtig wie Sequenzunterschiede scheinen auch andere Variationen zu sein, insbesondere Variationen in der Anzahl Kopien von bestimmten Genomabschnitten („copy number variations“, CNV). Mit all diesen Unterschieden ist klar, dass es keine zwei Menschen gibt, die sich genetisch gleichen. Selbst eineiige Zwillinge weisen Unterschiede auf, die z. B. in frühen Phasen der Embryonalentwicklung auftreten. Darüber hinaus ist das Genom einer ständigen Veränderung durch Modifikationen unterzogen, die teilweise durch Umwelteinflüsse getriggert werden. Diese epigenetischen Veränderungen haben entscheidende Einflüsse auf unser Genom und erhöhen damit ganz wesentlich die mögliche Variabilität. Diese enorme Breite der Variabilität erklärt auch, warum genetische Tests nie $100 \%$ prädiktiv sein können, sondern praktisch immer mit einer großen Variationsbreite verhaftet sind. Zu viele Faktoren beeinflussen die Entstehung und den Verlauf von Krankheit und Gesundheit, als dass diese mit den derzeit zur Verfü- gung stehenden Tests vollständig vorhergesagt werden könnten.

Die Umsetzung dieses mittlerweile enormen Wissens über Genfunktion, $\mathrm{Mu}$ tationspathologie und sowie Einsatz und Durchführung von Gentests erfordert Expertise, wie sie ganz wesentlich die Humangenetik leisten kann. Zugleich muss aber auch ein Verständnis über die Funktionen und grundlegenden Verfahren der genetischen Analyse bei den Ärzten vorliegen, die sich häufig mit genetischen Erkrankungen beschäftigen. Natürlicherweise ist dies besonders bei Gynäkologen gegeben, die sich mit Familienplanung bzw. Pränataldiagnostik auseinander setzen. Ebenso ist die genetische Diagnostik aber zunehmend wichtig bei hereditären Krebserkrankungen, wie dem Brust- und Darmkrebs.

Weiterhin fordert das nun in Kraft getretene Gendiagnostikgesetz eine Qualifikation von der verantwortlichen ärztlichen Person, die genetische Untersuchungen veranlasst. Nach Vorliegen der Untersuchungsergebnisse soll eine genetische Beratung bei einem Arzt, „der sich für genetische Beratungen qualifiziert hat" angeboten werden. Eine prädiktive genetische Untersuchung darf nur durch Fachärzte für Humangenetik oder durch Ärzte, „die sich beim Erwerb einer Facharzt-, Schwerpunkt- oder Zusatzbezeichnung für genetische Untersuchungen im Rahmen ihres Fachgebietes qualifiziert haben", vorgenommen werden. Hier sind neue Fort- bzw. Weiterbildungsinhalte zu definieren.

\section{() Genetische Diagnostik ist immer mit Aufklärung und Beratung zu verbinden}

In den Beiträgen dieser Ausgabe möchten wir einen Überblick über die derzeit angewendeten Verfahren der genetischen Diagnostik geben und sie zugleich in Zusammenhang mit der genetischen Beratung bzw. klinisch-genetischen Diagnostik stellen. Die derzeit verwendeten diagnostischen Verfahren der $\mathrm{Hu}$ mangenetik lassen sich einteilen in die klassische Zytogenetik, die molekulare DNA-Diagnostik und neuere Verfahren der komparativen Genomhybridisierung, wie die Array-CGH. Die verschie- denen Anwendungsbereiche, Möglichkeiten und Grenzen werden in den einzelnen Beiträgen dargestellt. Genetische Diagnostik sollte immer mit einer Aufklärung und Beratung des Patienten verbunden sein. Im Beitrag „Genetische Beratung" werden die Grundlagen für die Beratung und eine klinisch-genetische Diagnostik dargestellt.

\section{Ausblick}

Die Humangenetik hat über die letzten Jahre eine enorme Wandlung vollzogen, die sich aus technologischen Erneuerungen und einer vertieften Kenntnis über den Aufbau und die Funktion unseres Genoms herleitet. Immer mehr Erkrankungen wurden einzelnen Gendefekten zugeordnet, so wird das Wissen über die Anatomie und Pathologie unseres Genoms dramatisch erweitert. Für mehrere tausend Erkrankungen stehen heute genetische Tests zur Verfügung, die Zahl steigt weiter. Allerdings sind für diese Tests immer Verdachtsdiagnosen notwendig, sie können also nicht als genomweites Screening auf Veränderungen eingesetzt werden.

Durch die Array-CGH wurde es erstmals möglich, hochauflösend das gesamte Genom auf kleine und kleinste Veränderungen (Deletionen/Duplikationen) zu untersuchen. Im Prinzip war diese Untersuchung auch mit einer zytogenetischen Analyse möglich, doch die Array-CGH bietet eine wesentlich höhere Auflösung und zudem die Möglichkeit der Automatisierung. Mittlerweile verwenden wir Array-CGH mit sehr hoher Auflösung, mit der alle bekannten Deletionen/Duplikationen sicher identifiziert werden können. Während diese Verfahren im postnatalen Bereich, insbesondere bei der Diagnostik von unbekannten Krankheitsbildern/Syndromen, einen Durchbruch darstellt, wirft diese neue Form der Diagnostik besondere Fragen in der Pränataldiagnostik auf. Jede Diagnostik, die durch ihre hohe Auflösung viel identifiziert, wird auch viele Veränderungen nachweisen, deren Effekt nicht eindeutig ist bzw. die nach dem momentanen Stand des Wissens nicht sicher interpretierbar sind. Insbesondere im Pränatalbereich sind solche 
Veränderungen extrem schwer zu vermitteln, da sie doch Entscheidungen verlangen, die auf Grund der Informationslage gar nicht möglich sind. $\mathrm{Ob}$ und wie die Array-CGH in Zukunft für pränatale Zwecke eingesetzt wird, bleibt abzuwarten, eine Veränderung der Möglichkeiten in diesem Bereich stellt sich aber ohne Frage dar. Auch jetzt kann sie schon angewendet werden, wenn z. B. komplexe schwere Fehlbildungen vorliegen, die weiter genetisch abgeklärt werden sollen.

\section{(7) Folgen der \\ Genomsequenzierung weisen weit über die Genetik hinaus}

Die Entwicklung neuer Methoden der Sequenzierung ermöglicht es heute, ganze Genome bzw. große Teile des Genoms auf einmal zu sequenzieren, ohne das einzelne Abschnitte vorher amplifiziert und dann einzeln analysiert werden müssen. Die NGS (Next Generation Sequencing)-Technologie hat die molekulare Analyse des Genoms revolutioniert und wird ohne Zweifel großen Einfluss auf die weitere genetische Diagnostik haben. Schon jetzt ist es möglich, mit dieser Technologie alle transkribierten Gene auf einmal zu sequenzieren und $\mathrm{zu}$ analysieren. Es ist zu erwarten, dass diese Technologie für bestimmte Genpanels, wie sie z. B. bei manchen Erkrankungsgruppen vorliegen, zum Einsatz kommt und dann ganze Gengruppen auf einmal analysiert werden können. Die genetische Diagnostik wird durch diese Verfahren ohne Zweifel einen weiteren Schub erhalten, die sich auch auf die individuelle Familienplanung bzw. Prognose von bestimmten Erkrankungen wie z. B. Krebs, auswirken wird. Wie in $\mathrm{Zu}-$ kunft mit diesen Technologien im medizinischen Bereich umgegangen wird, und ob eine Genomsequenzierung zum medizinischen Alltag gehören wird, ist derzeit noch nicht absehbar. Sicher erscheint jedoch, dass sich hier gewaltige Veränderungen aufzeigen, die weit über die Genetik hinaus ausstrahlen werden.

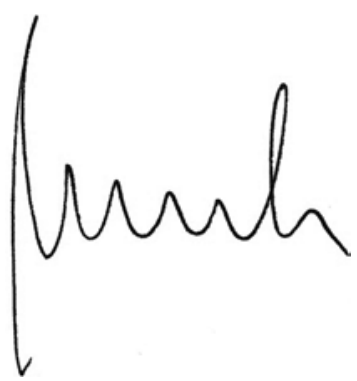

Prof. Dr. Stefan Mundlos

\section{Korrespondenzadresse}

Prof. Dr. S. Mundlos

Institut für Medizinische Genetik, Charitè, Universitätsmedizin Berlin, Campus Virchow Augustenburger Platz 1, 13353 Berlin stefan.mundlos@charite.de

\section{Hier steht eine Anzeige.}

\section{Springer}

